\section{Assessing the impact of airborne outreach to build clinical capacity in rural Botswana}

\author{
Michael J. A. Reid,,,2 Brianna L. Kirk ${ }^{3}$ \\ 'Botswana UPenn Partnership, Botswana; \\ 2University of Pennsylvania, \\ Pennsylvania, USA
}

${ }^{3}$ Baylor College of Medicine, Texas, USA

\section{Abstract}

There is a paucity of research demonstrating how best to address inequalities in health and access to specialist care faced by rural disadvantaged populations in high HIV-prevalent settings in Sub Saharan Africa. Delivering equitable and cost-effective specialist clinical services in many parts of Africa is challenging, given human resource shortages, poor transport infrastructure and competing health priorities. In this report we describe how an airborne outreach program to provide HIV services to high HIV burden health facilities in rural Botswana has been an important catalyst for improving specialist service delivery across the spectrum of clinical care. The success of Botswana's airborne program is a consequence of many country-specific determinants as well as external funding support. We argue that lessons learned from the experience in Botswana are normative for other African settings. Specialist medical airborne outreach to rural hospitals can improve access to and quality of care, when part of a multifaceted, multidisciplinary intervention. Furthermore, we demonstrate how an HIV funded program can be a vehicle for enhanced access to essential sub-specialist clinicians in rural Botswana.

\section{Introduction}

Despite considerable investment in HIV programs in Africa over the last decade, little is known about whether funding for HIV care and treatment services has influenced access to and quality of other health services across the continent. ${ }^{1}$ Notably, there is little data describing whether HIV funding has improved access to non-HIV specialty services for rural communities in Africa. While evidence from highincome countries has demonstrated that rural outreach programs can improve health coverage and outcomes of non-HIV related diseases, ${ }^{2,3}$ it is less clear whether HIV-specific outreach programs have affected access to non-HIV services in rural settings in Africa. In this report we describe how airborne outreach to high HIV burden facilities has enhanced access to both HIV and non-HIV services in rural Botswana. Furthermore, we assess the applicability of airborne outreach to other settings in sub Saharan Africa, where both infrastructure and resources are more constrained. Finally we review the role of airborne outreach in Botswana as a catalyst for health system improvement.

In most African settings, clinical care is provided by clinical officers or general practitioners, with minimal access to sub-specialist services. ${ }^{4}$ Where available, specialist medical practitioners usually consult in outpatient areas of major hospital facilities or large metropolitan cities. Unfortunately, across southern Africa, access to specialist services is very limited for those living in remote and rural settings, as they have to travel hundreds of kilometers to reach referral centers. Compared to urban populations, people living in rural and remote areas tend to have worse health and less access to medical care. ${ }^{5}$ Limited healthcare infrastructure and depleted workforces that are commonplace in many African settings are combined with an approach to healthcare that is largely curative and episodic in nature. ${ }^{6}$ Most health encounters are brief and solely for management of acute symptoms, failing to address chronic diseases or preventative healthcare. Limited medical record systems, inability to track missed visits, and stock outs of laboratory reagents and medications also hamper the effectiveness of existing specialist services. ${ }^{7}$ This fractured approach is clearly suboptimal in the face of HIV disease, as well as the management of other chronic diseases, that require access to specialty medical services. In addition, as life expectancy normalizes for African HIV-infected patients on highly active antiretroviral therapy, the burden of adult chronic diseases in African countries is likely to increase among people living with HIV. ${ }^{8}$

The benefits of specialist care are particularly important in the setting of the HIV epidemic, which has had a devastating impact on overburdened health systems across Africa. ${ }^{9}$ The effective management of HIV disease as a chronic condition necessitates the provision of specialist services to provide training, mentoring and clinical oversight for health care workers managing patients. For these reasons, HIVspecific outreach has become a vital component of HIV programs over the last ten years as countries with high HIV sero-prevalence have recognized the need to provide HIV-specific mentorship commensurate with decentralization of antiretroviral services to rural health care settings. ${ }^{10,11}$

Unfortunately, provision of specialist HIV services in rural settings is challenging to implement in many African countries for
Correspondence: Michael J. A. Reid, P0 Box AC 157, ACH Riverwalk, Gaborone, Botswana. Tel: +267.724.78777 - Fax: +267.3593.2267

E-mail: michael.j.a.reid@gmail.com

Key words: HIV, outreach, airborne, health system strengthening.

Received for publication: 15 May 2013

Revision received: Not requested.

Accepted for publication: 31 August 2013.

This work is licensed under a Creative Commons Attribution NonCommercial 3.0 License (CC BYNC 3.0).

(C) Copyright M.J.A. Reid and B.L. Kirk, 2013

Licensee PAGEPress, Italy

Journal of Public Health in Africa 2013; 4:e11

doi:10.4081/jphia.2013.e11

numerous reasons. HIV outreach programs are generally expensive and assume that specialists are available to participate. Effective mentoring requires both time and consistent mentees, an ongoing challenge among overburdened health systems experiencing constant turnover. Furthermore, outreach programs incur numerous opportunity costs associated with taking specialists out of their main practice. For these reasons, many remote, rural communities across southern Africa are unable to access such outreach programs.

Botswana has one of the world's highest HIV prevalence rates with an estimated $25 \%$ among adults aged 15-49 and a proportionally large number of HIV-infected children. In the face of this devastating epidemic, the country has established one of Africa's most progressive HIV care and treatment programs. By 2010 Botswana achieved near universal access: 93\% of all eligible patients had been initiated on antiretrovirals (ARVs). The speed and scope of Botswana's ARV scale up has been attributed to a number of factors: significant donor investment, good governance, high quality HIV-specific training ${ }^{12}$ and effective mentorship programs to facilities. ${ }^{13}$

Supporting HIV scale up to the most remote parts of Botswana has proven to be a formidable challenge. Large distances and human resource challenges are significant obstacles to HIV care and treatment in Botswana. While the majority of the country lives in the two major cities, Francistown and Gaborone, remote communities in the most rural parts of the country have the highest HIV prevalence. ${ }^{14}$ To address these challenges the Botswana airborne initiative was launched in 2007 in partnership with Airborne Lifeline Foundation (ALF), transporting specialists to several of the most remote, high burden HIV clinics. ALF is funded by the United States government, through the President's Emergency Plan for 
AIDS Relief (PEPFAR) ${ }^{15}$ in collaboration with the Botswana Ministry of Health $(\mathrm{MoH})$, to deliver the specialist HIV care and treatment services in remote, high volume health facilities. ALF coordinates flights once a month to each supported facility, liaising with the main teaching hospital in Gaborone, to ensure that specialists from different disciplines attend on these flights. As well as providing HIV specialist support, ALF's program also facilitates provision of non-HIV sub-specialty services, such as orthopaedics and otolaryngology, to the same facilities. ALF also liaises with the MoH's Central Medical Supplies Department, to ensure that all flights deliver medical supplies as required. While most supplies are still delivered by road, the flights provide an opportunity for the MoH to respond to stock-outs when necessary.

Facilities supported through airborne outreach are all sites that had limited access to any of these services before introduction of the flying outreach service and instead referred patients to one of two tertiary referral centers, several hundred kilometers away. Facilities are determined by $\mathrm{MoH}$, as high volume sites that warrant access to specialist services. Prerequisite to flights, all facilities must also be serviced by a basic runway, either gravel or asphalt. Since outreach planes are 8 seater King Air 200 planes, all runways are minimum $1000 \mathrm{~m}$ in length, administered by the Botswana civil aviation authority.

\section{Materials and Methods}

In order to assess the scope and impact of ALF's program in Botswana we performed a retrospective analysis of all outreach flights flown between July 2011 and June 2012. Specialist outreach flights were defined as planned visits by specialist-trained medical practitioners to government-determined high volume hospitals in rural locations. Supported facilities were all assigned by the Ministry of Health, and determined to be high HIV-burden sites, too remote to be supported by road-based outreach services.

Recorded details from flights to these facilities included the number of health care providers participating on each visit and the number of individual patient encounters, as reported by outreach clinicians, on each trip. From March 2012 onwards information on HIV status of all patients seen on outreach was also reported.

Access to care was described in terms of sub-specialist clinical services rendered at these sites and the number of clinical encounters performed by sub-specialty clinicians. These outcomes have been used widely as metrics to assess impact of outreach programs. ${ }^{16}$ Since the analysis included only routinely collected data, Institutional Review Board approval was not required. Descriptive statistical analysis was performed using Microsoft Excel (Microsoft, 2011).

\section{Results}

During 12 months, between July 2011 and June 2012, airborne outreach provided essential clinical services to five high volume, rural health facilities: Ghanzi, Hukuntsi, Kasane, Maun and Tsabong (Figure 1). The characteristics of these sites are reported in Table 1. $14,17,18$ Supported facilities serve communities with HIV prevalence estimated at between
11.8\% (Hukuntsi) and 23.0\% (Kasane). ${ }^{14}$ Facilities were between $509 \mathrm{~km}$ (Hukuntsi) and $933 \mathrm{~km}$ (Kasane) away from the supporting tertiary referral center, in Gaborone.

Our review of Botswana's airborne outreach program support was notable for the following findings. Firstly, airborne outreach provided access to specialist services to primary care doctors working at supported facilities. Over a twelve-month period, airborne outreach facilitated 5032 patient referrals to specialist clinicians. An average of 90 patient encounters occurred at each site per visit (Table 2).

Secondly airborne outreach improved HIV training and mentorship at supported sites. Prior to airborne outreach, none of the sup-

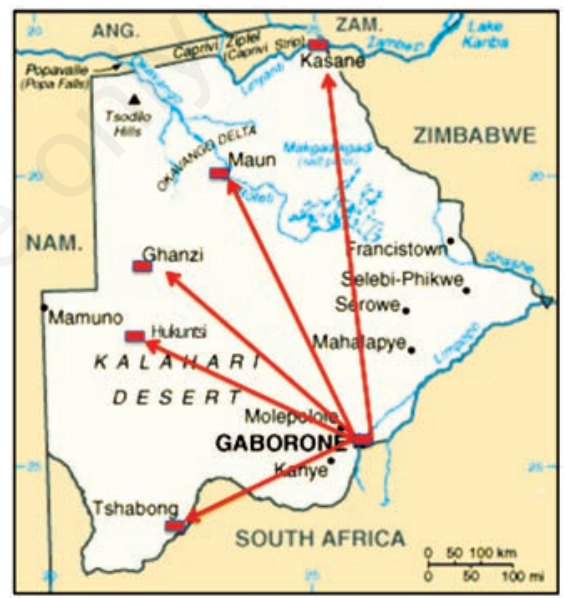

Figure 1. Map of Botswana with flight paths (image courtesy of Central Intelligence Agency website, available from: https://www.cia.gov/library/publications Accessed: September 11, 2013).

Table 1. Outreach destination characteristics.

\begin{tabular}{|c|c|c|c|c|c|}
\hline Facility & City population ${ }^{17}$ & $\begin{array}{l}\text { Catchment } \\
\text { population }^{17}\end{array}$ & $\begin{array}{l}\text { Estimated HIV } \\
\text { prevalence }^{14}\end{array}$ & $\begin{array}{l}\text { Distance from } \\
\text { Gaborone }(\mathrm{km})\end{array}$ & $\begin{array}{c}\text { Manned airstrip } \\
(\mathrm{Y} / \mathrm{N})^{18}\end{array}$ \\
\hline Hukuntsi & 3807 & 20,476 & $11.8 \%$ & 509 & $\mathrm{~N}$ \\
\hline Ghanzi & 12,267 & 43,095 & $13.5 \%$ & 543 & $\mathrm{Y}$ \\
\hline Tsabong & 7869 & 30,106 & $19.1 \%$ & 514 & $\mathrm{~N}$ \\
\hline Maun & 55,784 & 152,284 & $19.8 \%$ & 577 & $\mathrm{Y}$ \\
\hline Kasane & 9127 & 23,347 & $23 \%$ & 933 & $\mathrm{Y}$ \\
\hline
\end{tabular}

$\mathrm{Y}$, yes; $\mathrm{N}$, no.

Table 2. Total no. of patients encounters (August 2011- July 2012) at each site.

\begin{tabular}{lccc} 
Facility & No. of visits & Total no. of patients encounters & Average no. of patients encounters/visit \\
Hukuntsi & 11 & 1089 & 99 \\
Ghanzi & 12 & 710 & 59 \\
\hline Tsabong & 11 & 1162 & 106 \\
Maun & 11 & 1437 & 131 \\
\hline Kasane & 11 & 634 & 57 \\
Total & 56 & 5032 & 90 \\
\hline
\end{tabular}


ported facilities had regular access to HIV specialists. The airborne program ensured that HIV specialists, providing paediatric and adult services, attended every facility. These specialists provided HIV-specific mentoring and preceptorship. They also provided clinical care for patients with complicated HIV-specific problems. Between April and June 2012, 20\% $(n=390)$ of all of patients seen by specialists on outreach involved HIV positive patients (Table 3). Thirdly, airborne outreach improved access to a broad range of other specialty services, including but not limited to, oncological, psychiatric and neurosurgical expertise. Between April and June 2012, orthopedic surgeons and otolaryngologists, for example, both saw an average of 48 and 59 patients per outreach visit respectively, demonstrating the extraordinary demand for their services at supported facilities (Table 4). Most HIV specialists are either internal medicine or paediatric specialists as well, seeing non-HIV related medical and paediatric referrals in addition to HIVinfected patients.

Finally, our analysis demonstrates that airborne outreach facilitated multidisciplinary capacity building initiatives. Flights were also attended by numerous allied health professionals, including nurses, physiotherapists, dieticians, and psychologists. These health professionals supported numerous activities to build capacity of local doctors and nurses, running education and counseling programs, as well as providing expert clinical care.

\section{Discussion}

We report on the successful implementation of an airborne outreach program to support high burden health facilities in rural Botswana. We believe that this is the first report describing how a PEPFAR funded airborne outreach to deliver HIV services can be leveraged to improve health access across the spectrum of care. While we acknowledge that the small scale of the program limits its applicability to other settings, we believe that the implementation of this program offers important insights that can inform how and when airborne outreach services are employed elsewhere in Africa.

Specifically, our analysis demonstrates how HIV-specific outreach increased access to other sub-specialty services in rural communities and provides concrete evidence that HIVspecific funding can enhance provision of nonHIV services in rural Africa, when provided as part of a coordinated multi-disciplinary program. We believe that the successes of Botswana's airborne outreach program are based on several key tenets.

Firstly, our report demonstrates that airborne outreach is an effective and expedient strategy for providing HIV specialist care in remote, high HIV prevalent settings. Over a 3month period, specialists were able to undertake over one thousand HIV-specific clinical encounters. Because of the remoteness of the clinics visited, it is unlikely that many of these encounters would have otherwise occurred; patients that would have had to travel long distances to see HIV specialists at tertiary referral centers could be seen locally. Outreach ensured that the best-trained HIV specialists in the country were available to support the management of complicated patients at each of the outreach facilities. Patients with complex co-morbidities, co-infections, HIV treatment failure and AIDS associated malignancies were all able to receive care from specialists that they would not otherwise have had access to. Outreach visits also facilitated HIVspecific training and mentorship for doctors and nurses working at the rural clinics.

Secondly, our report demonstrates that this airborne program was an important means of capacity building to improve clinical capacity across the spectrum of care. Outreach has provided opportunities for a wide range of teaching opportunities including joint consultations, educational sessions, and precepted outpatient visits, seminars and case-conferences at sites that had not previously received regular teaching. This component of the program was a pre-requisite of PEPFAR funding. However, it is consistent with existing research demonstrating that outreach that involves little more than a shift in the location of the consultation, confers little benefit limited to measures of convenience and patient satisfaction. The impact of capacity building initiatives at sites supported by the airborne pro-

Table 3. No. of patients encounters disaggregated by HIV status (April-June 2012).

\begin{tabular}{lccc}
\hline Facility & No. of visits & No. of patient encounters & No. HIV patient encounters (\%total) \\
Hukuntsi & 3 & 232 & $79(25)$ \\
Ghanzi & 3 & 231 & $62(28)$ \\
\hline Tsabong & 3 & 321 & $64(20)$ \\
Maun & 3 & 321 & $71(22)$ \\
\hline Kasane & 3 & 269 & $26(10)$ \\
Total & 15 & 1053 & $302(28)$ \\
\hline
\end{tabular}

Table 4. No. of patient encounters disaggregated by clinical specialty.

\begin{tabular}{lcc} 
Specialty & $\begin{array}{c}\text { No. of patients encounters } \\
\text { by sub-specialists (April-June 2012) } \\
(\% \text { all clinician encounters) }\end{array}$ & $\begin{array}{c}\text { Average no. of patient encounters } \\
\text { by sulb-specialists at each clinic }\end{array}$ \\
Orthopaedics & $298(29)$ & 59 \\
ENT & $95(8)$ & 48 \\
\hline Oncology & $47(4)$ & 8 \\
Neurosugery & $42(4)$ & 14 \\
\hline Psychiatry & $97(8)$ & 13 \\
Internal medicine & $190(18)$ & 24 \\
\hline Pediatrics & $307(29)$ & 23 \\
Total & 1034 & 23 \\
\hline
\end{tabular}

ENT, Ear, Nose, Throat Department. 
gram have been described elsewhere. ${ }^{13,19}$

Thirdly, this report demonstrates that airborne outreach is a successful multidisciplinary intervention. Specialists from a variety of disciplines participated on outreach flights. Most flights included clinicians from at least 3 different medical sub-specialties as well as numerous health care professionals, from radiology technicians to physiotherapists and psychologists. In our experience outreach was most successful when delivered as part of a multi-disciplinary and multifaceted approach. Furthermore, we speculate that extra costs of airborne outreach are at least partly offset by reduced costs for the patient and greater costeffectiveness of a multi-disciplinary approach.

A crucial component of the airborne outreach program was that it enhanced access to clinical services to disenfranchised rural communities. Such rural communities have the most to gain from any outreach program in terms of client outcomes. The fact that most specialist outreach programs in Africa are disproportionately concentrated around major urban centers results in access inequities and can compound the fact that rural populations are usually less healthy. ${ }^{16}$ While we acknowledge that a key limitation of our study is that it is a post-hoc analysis of services already established for which evaluation had not been planned prior to implementing the intervention, we argue that it provides persuasive data illustrating that airborne outreach improves access to specialist services for the most remote and rural communities. Before the airborne program started, the five sites included in our analysis sites did not receive any regular specialist outreach; after the program started, they were all guaranteed regular access to specialist services. We believe that our data demonstrates that when resources are available, airborne outreach is feasible in an African setting. While there is existing data from high-income countries that airborne outreach is an effective intervention ${ }^{20-22}$ there has been minimal operational research describing the feasibility of airborne programs in subSaharan African settings. There are reports that airborne outreach by surgical teams have been successful in Kenya, ${ }^{22}$ Zimbabwe, ${ }^{23}$ and Uganda. ${ }^{24}$ However, we believe this is the first report describing how multidisciplinary outreach is feasible and effective in an African context. Nevertheless, we stress that this feasibility is entirely predicated on donor funding and that given the financial constraints affecting most health systems in southern Africa we recognize that without external donor support, programs such as the one we describe are not replicable in other SSA settings.

We acknowledge that airborne outreach has been successful in Botswana because of many contextual factors: the population is small, infrastructure is already in place and the gov- ernment has been very supportive of the program. There are clear limitations and potential harms associated with establishing an airborne specialist outreach programs. We acknowledge that such programs are very expensive. Outreach flight costs are considerable, especially when measured against standard outreach by motor vehicle. Furthermore, the program has its own running costs and indirect expenses. In our experience, the kind of clinical practice employed by specialists is generally more expensive than that of untrained medical officers. Specialist physicians are more likely to prescribe expensive drugs and suggest more diagnostic investigations than general practitioners. While these are invariably warranted they incur increased costs for the visited sites and may not always reduce the number of referrals to tertiary referral centers. Given the extraordinary financial constraints faced by the Botswana government, it is unclear whether the airborne program will be sustained when PEPFAR funding is withdrawn in its entirety. We argue that a formal cost-effectiveness evaluation is necessary to determine whether the program is expensive as it appears, when weighed against savings as a consequence of improved clinical outcomes and reduced numbers of referrals to Botswana's referral hospitals.

Outreach to rural sites is essential to address inequity between rural and urban services. However, outreach may compromise care at urban facilities if rural sites receive care at the expense of those urban facilities. Furthermore, given the traveling time, airborne outreach may be an inefficient use of specialists' time when those specialists could be consulting in their primary practice at urban facilities. While we acknowledge that all of these limitations are important, we argue that the extra costs, both time and money, may be partly offset by reduced costs for the patient and the greater cost-effectiveness of multifaceted interventions.

Airborne outreach plays an important role in the delivery of clinical services in Botswana. The existing program offers remarkable opportunities to improve clinical outcomes to rural communities across the country. However, its continued success is predicated on external donor support. As PEPFAR funding across the region diminishes, we suggest that the following steps are necessary to ensure that clinical impact is not compromised by competing public priorities and financial expediencies. Firstly, we argue that an HIV-specific airborne outreach in Botswana is advantageous when delivered as part of a multifaceted intervention. Assessments of outreach programs in other settings have demonstrated that multidisciplinary outreach reduces costs and also reduces the number of patient referrals to tertiary centers and unnecessary duplicated tests. ${ }^{16}$ Given that the airborne outreach program in Botswana is funded entirely through donor support and in light of the significant constraints on health care systems in many parts of Africa, there is considerable debate about whether airborne programs are a viable solution to the challenges of inequitable access in rural, African settings. Other outreach interventions, including web-based mentorship ${ }^{25}$ the use of real time Voice Over internet Protocol videoconferencing and $\mathrm{m}$ health programs ${ }^{26}$ offer effective low cost alternatives for providing mentorship to clinicians in remote settings. Such solutions are particularly advantageous given increasing Internet access via cellular networks across the continent. ${ }^{27}$ A multifaceted outreach program, combining remote support via web-based platforms and airborne support may represent a viable long-term option, as external funding for outreach diminishes.

We believe that the successes of programs such as ALF's airborne program are predicated on finding long-term funding options. One potential solution is that of public-private partnerships (PPPs). PPPs have been integral to Botswana's response to the HIV epidemic. Established in 2000, the African Comprehensive HIV/AIDS Partnerships (ACHAP) is an example of a country-led, public-private partnership between the Government of Botswana, the Bill \& Melinda Gates Foundation, and MSD/Merck Company Foundation. This PPP has been instrumental in Botswana's national response to HIV/AIDS, supporting scale up of antiretrovirals through provision of financial and technical support to the Government of Botswana as well as the civil society sector through some Non-Governmental Organizations. ${ }^{28}$ ACHAP offers a successful model of an effective and mutually beneficial PPP. Given that commercial airlines fly to several rural communities in northern Botswana, there is considerable opportunity for the Ministry of Health to replicate the successes of ACHAP in the context of airborne outreach. While such collaboration may not be possible in other African settings given lack of airport infrastructure and prohibitive costs for private airborne travel to rural communities, PPPs to support airborne outreach represents a tangible sustainable financial model in Botswana.

Finally, we suggest that operational research efforts must be a key priority to assess the impact of PEPFAR funded programs such as the one described in this report. Little is known about how HIV-related funding and the development of institutional resources to support HIV services in Botswana has influenced the access to and the quality of other health services. Clinics and hospitals across the country have been overwhelmed with the large number of patients crowding waiting rooms and hospital beds, with a substantial 
proportion with underlying HIV infection and with HIV-related complications, such as pneumonia, tuberculosis, diarrhea, wasting and meningitis. The provision of ART and the establishment of airborne outreach programs to rural facilities have decongested medical clinics, emergency services and tertiary referral facilities. However, the ultimate effect of Botswana's airborne outreach program will need to be rigorously evaluated. It is important that further research is focused on determining whether airborne outreach is i) clinically effective and ii) cost-effective.

\section{Clinical impact research}

Rigorous research to assess how airborne outreach improves clinical outcomes is vital. The effect on other programmatic frameworks across the health system should also be evaluated. Parameters of interest include monitoring the utilization of laboratory services and access to non-HIV services provided on outreach flights.

\section{Cost-effectiveness analysis}

While airborne outreach offers the clear benefits for in-person mentorship and patient contact, the programmatic costs are considerable. We believe that airborne outreach may be cost-effective when expensive transport costs are weighed against the direct and indirect costs of not providing specialist clinical services to remote areas. Further research is needed to determine if this is the case.

\section{Conclusions}

Airborne outreach to rural facilities in Botswana is necessary to support continuing HIV care at these remote, high burden facilities. Since multi-disciplinary non-HIV specialist services were also provided, the program illustrates how HIV-funded interventions can be effectively leveraged to improve access and equity to health care in remote settings in Africa. Furthermore, by fostering strong links between rural facilities and tertiary referral services, airborne outreach is an important tool for building clinical capacity. More research is warranted to determine the clinical impact and the cost-effectiveness of this service.

\section{References}

1. Duber HC, Coates TJ, Szekeras G, et al. Is there an association between PEPFAR funding and improvement in national health indicators in Africa? A retrospective study. J Int AIDS Soc 2010;13:21.

2. Chopra M, Sharkey A, Dalmiya N, et al. Strategies to improve health coverage and narrow the equity gap in child survival, health, and nutrition. Lancet 2012;380: 1331-40.

3. Eaton J, McCay L, Semrau M, et al. Scale up of services for mental health in lowincome and middle-income countries. Lancet 2011;378:1592-603.

4. Mullan F, Frehywot S. Non-physician clinicians in 47 sub-Saharan African countries. Lancet 2007;370:2158-63.

5. Powell J. Systematic review of outreach clinics in primary care in the UK. J Health Serv Res Policy 2002;7:177-83.

6. Assal JP. Revisiting the approach to treatment of long-term illness: from the acute to the chronic state. A need for educational and managerial skills for long-term follow-up. Patient Educat Counsel 1999;37: 99-111.

7. Loewenson R, McCoy D. Access to antiretroviral treatment in Africa. BMJ 2004; 328:241-2.

8. Johnson LF, Mossong J, Dorrington RE, et al. Life expectancies of South african adults starting antiretroviral treatment: collaborative analysis of cohort studies. PLoS Med 2013;10:e1001418.

9. El-Sadr WM, Abrams EJ. Scale-up of HIV care and treatment: can it transform healthcare services in resource-limited settings? Aids 2007;21:S65-70.

10. Bedelu M, Ford N, Hilderbrand K, Reuter $\mathrm{H}$. Implementing antiretroviral therapy in rural communities: the Lusikisiki model of decentralized HIV/AIDS care. J Infect Dis 2007;196:S464-8.

11. Loubiere S, Boyer S, Protopopescu C, et al. Decentralization of HIV care in Cameroon: increased access to antiretroviral treatment and associated persistent barriers. Health Policy 2009;92:165-73.

12. Bussmann C, Rotz P, Ndwapi N, et al. Strengthening healthcare capacity through a responsive, country-specific, training standard: the KITSO AIDS training program's support of Botswana's national antiretroviral therapy rollout. Open AIDS J 2008;2:10-6.

13. Workneh G, Scherzer L, Kirk B, et al. Evaluation of the effectiveness of an outreach clinical mentoring programme in support of paediatric HIV care scale-up in Botswana. AIDS Care 2013;25:11-9.

14. Botswana Go. Botswana Aids Impact Survey III (BAIS III) 2008 Preliminary Result. Gaborone: Central Statistics Office; 2008.

15. Creswell J, Jaramillo E, Lonnroth K, et al.
Tuberculosis and poverty: what is being done. Int J Tuberc Lung Dis 2011;15:431-2.

16. Gruen RL, Weeramanthri TS, Knight SE, Bailie RS. Specialist outreach clinics in primary care and rural hospital settings. Cochrane Database Syst Rev 2004(1): CD003798.

17. Central Statistics Office. Census Botswana 2011. Available from: http://www.cso. gov.bw/index.php?option=com_content1\&id=2\&site=census Accessed: 24 April 2013.

18. Civil Aviation Authority of Botswana. Ground handlers; 2013. Available from: http://www.caab.co.bw/caab-content.php? cid=134 Accessed: 24 April 2013.

19. Wester CW, Kim S, Bussmann H, et al. Initial response to highly active antiretroviral therapy in HIV-1C-infected adults in a public sector treatment program in Botswana. J Acquir Immune Defic Syndr 2005;40:336-43.

20. Baker JW, Buttini MJ. The flying obstetric and gynaecology service in rural Queensland: its first two years. Med J Aust 1991;154:578-82.

21. Gruen RL, Bailie RS, d'Abbs PH, et al. Improving access to specialist care for remote Aboriginal communities: evaluation of a specialist outreach service. Med J Aust 2001;174:507-11.

22. Kapur A, Harries AD. The double burden of diabetes and tuberculosis - Public health implications. Diabet Res Clin Pract 2013. [Epub ahead of print].

23. Baker MA, Lin HH, Chang HY, Murray MB. The risk of tuberculosis disease among persons with diabetes mellitus: a prospective cohort study. Clin Infect Dis 2012;54:818-25.

24. Nissapatorn V, Kuppusamy I, Jamaiah I, et al. Tuberculosis in diabetic patients: a clinical perspective. Southeast Asian J Trop Med Public Health 2005;36:213-20.

25. Reid MJ, Flam R, Tsiouris F. New models for medical education: web-based conferencing to support HIV training in SubSaharan Africa. Telemed J E Health 2012; 18:565-9.

26. Fischer MK, Kayembe MK, Scheer AJ, et al. Establishing telepathology in Africa: lessons from Botswana. J Am Acad Dermatol 2011;64:986-7.

27. Meso P, Mbarika VW, Sood SP. An overview of potential factors for effective telemedicine transfer to Sub-Saharan Africa. IEEE Trans Inf Technol Biomed 2009;13:734-9.

28. Ramiah I, Reich MR. Building effective public-private partnerships: experiences and lessons from the African Comprehensive HIV/AIDS Partnerships (ACHAP). Soc Sci Med 2006;63:397-408. 